\title{
Análise descritiva quantitativa do palmito de pupunheira
}

\author{
Marta Regina VERRUMA-BERNARDI ${ }^{1}$, Carla Wilma Santos de MORAES $^{2}$, Carla Almeida MACHADO², \\ Shizuko KAJISHIMA², Ester de Queirós COSTA²
}

\section{RESUMO}

Este estudo foi conduzido para descrever o perfil sensorial do palmito de pupunha. A análise descritiva quantitativa (ADQ) foi utilizada para descrever os atributos sensoriais relacionados à aparência, aroma, e textura de três marcas comerciais de palmito de pupunha. As amostras foram avaliadas por uma equipe selecionada com onze provadores e foram definidos nove atributos sensoriais: cor amarela, aparência uniforme e úmida, aroma e sabor não característicos, sabor acido, residual acido e textura macia. Houve diferenças significativas em seis atributos entre as três marcas testadas. Os provadores descreveram os palmitos de pupunha como: cor amarela clara, aparência uniforme e úmida, aroma e sabor não característicos e sabor ácido com valores intermediários, residual acido e amargor final com valores de pouco e textura muito macia a uma textura intermediária.

PALAVRAS-CHAVE: Bactris gasipaes Kunth, Análise sensorial, Atributos sensoriais, Provadores treinados.

\section{Quantitative descriptive analysis of pejibaye palm heart}

\section{ABSTRACT}

This research was carried out to establish the sensory profile of pejibaye palm heart. The quantitative descriptive analysis was used to establish the sensory attributes related to appearance, flavor, taste and texture of tree commercial marks of pejibaye palm heart. These samples were evaluated by eleven trained panelists who described nine sensory attributes: yellow color, uniform and humid appearance, non-characteristic aroma and flavor, acid flavor, residual acid taste, and soft texture. There were significant differences in six attributes among the three tested marks. Panelists described the pejibaye palm heart as: light yellow color, uniform and humid appearance, non-characteristic aroma and flavor, acid flavor with medium values, acid residual taste and bitterness final taste from little values and high soft texture to an medium value texture.

KEYWORDS: Bactris gasipaes Kunth, Sensory analysis, Sensory attributes, Trained panelists.

'DTAiSER / Centro de Ciências Agrárias / UFSCar - Via Anhanguera, km 174 - Caixa Postal 153 CEP 13600-970 - Araras-SP. e-mail: verruma@cca.ufscar.br.

${ }^{2}$ Faculdade de Nutrição - UFF. Rua São Paulo, 30, $4^{\circ}$ andar, Campus Valonguinho, Centro, Niterói - RJ. 


\section{INTRODUÇÃOO}

Nos últimos anos, o cultivo da pupunheira (Bactris gasipaes Kunth) para a produção de palmito vem despertando o interesse de agricultores em todo o país, devido principalmente, a busca de novas opções de cultivo em substituição aos tradicionais, em virtude dos baixos preços alcançados por esses no mercado (Bovi, 1998). Além disso, as dificuldades encontradas no plantio racional do açaí e da juçara estão relacionadas ao tempo de maturação da cultura (em torno de quatro e oito anos, respectivamente), baixo rendimento de palmito por hectares/ano e a necessidade de sombreamento na fase inicial do plantio. A juçara apresenta ainda a inconveniência de ser monocaule (não perfilhar), necessitando de replantio após o corte (Araújo, 1996).

O esgotamento da oferta de matéria prima proveniente da coleta extrativa no centro sul, a intensificação da fiscalização de órgãos oficiais impedindo a devastação predatória, o custo da taxa de reflorestamento (próximo de $12 \%$ dos custos de industrialização), o alto preço do produto no mercado nacional e internacional, sendo que, no primeiro, a tonelada do produto líquido drenado vem sendo vendida a valores superiores a US\$ $4.000,00$ são fatores que vêm estimulando o investimento e produção racional para palmito industrializado.

Para Ferreira et al. (1990) a constante e crescente necessidade de suprimento de matéria-prima, para as indústrias no mercado nacional e internacional, tem despertado especial interesse em outros gêneros da família Palmae, particularmente os capazes de fornecer palmito de boa qualidade em curto prazo.

De acordo com Freitas \& Fugman (1990), do ponto de vista energético, o valor alimentício do palmito é muito baixo, porém pode ser considerado um bom fornecedor de minerais por conter sódio, potássio, manganês, cálcio, ferro, flúor, cobre, boro e silício.

De acordo com Pantoja et al. (2000), na Amazônia, devido a sua importância regional, o cultivo da pupunheira tem sido realizado em larga escala.

A pupunha se apresenta nesse contexto de diminuição de oferta e crescimento de consumo de palmito, como alternativa de cultivo racional. Devido ao potencial comercial do palmito de pupunha, muitos países latino-americanos estão investindo no seu cultivo e industrialização. $O$ interesse para cultivar a pupunha está aumentando fortemente nos últimos anos (Villachica, 1996), especialmente para a produção de palmito. Dois fatores que estão facilitando este aumento: a existência de um mercado a nível mundial e a disponibilidade de tecnologia para o cultivo e industrialização da pupunha para palmito.

O presente estudo visa estabelecer, através da Análise Descritiva Quantitativa, os atributos sensoriais que caracterizam o palmito de pupunha de três marcas existentes no mercado.

\section{MATERIAL E MÉTODOS}

Foram utilizadas amostras, apresentadas em toletes, de três marcas de palmito de pupunha, comercializadas no Rio de Janeiro, cuja composição química se encontra na Tabela 1.

Tabela 1 - Composição química dos palmitos estudados.

\begin{tabular}{ll}
\hline Componentes & Palmito de pupunha* \\
\hline Valor calórico & $30 \mathrm{Kcal}$ \\
Carboidratos & $0,4 \mathrm{~g}$ \\
Proteínas & $0,3 \mathrm{~g}$ \\
Gorduras totais & $0,1 \mathrm{~g}$ \\
Gorduras saturadas & - \\
Colesterol & - \\
Fibra alimentar & $0,1 \mathrm{~g}$ \\
\hline Sódio & $350 \mathrm{mg}$ \\
Ferro & - \\
Cálcio & - \\
Vitamina C & $3,6 \mathrm{mg}$ \\
\hline
\end{tabular}

Fonte: Rótulo dos produtos* (em 100 gramas), sendo a mesma composição nos três rótulos.

\section{ANÁLISE DESCRITIVA QUANTITATIVA}

Para a análise sensorial das amostras foi utilizado o método de Análise Descritiva Quantitativa (ADQ), adaptado da metodologia descrita por Stone \& Sidel (1985). Os testes foram realizados pela manhã no horário das 9:00 às 11:00 h no Laboratório de Alimentos e Dietética da Faculdade de Nutrição da Universidade Federal Fluminense.

\section{PRÉ-SELEÇÃO DA EQUIPE}

Inicialmente foram convidados para este estudo 20 provadores pré-selecionados levando-se em consideração o interesse e disponibilidade no período de realização da análise além do conhecimento prévio de análise sensorial.

\section{DESENVOLVIMENTO DA TERMINOLOGIA DESCRITIVA}

Cerca de 20 gramas de cada amostra de palmito, foram servidas à $10^{\circ} \mathrm{C}$ em pratos brancos descartáveis, codificadas com três letras e apresentadas aleatoriamente para a realização do teste. O levantamento de atributos foi feito através do método Rede - "Kelly's Repertory Grid Method" (Moskowitz, 1983). Foram realizadas três sessōes onde foram apresentadas duas amostras de palmito por vez ( $\mathrm{A}$ B $, \mathrm{A} \times \mathrm{C}, \mathrm{C} \times \mathrm{B})$, e foi solicitado que o provador anotasse as similaridades e as diferenças entre as amostras, utilizando ficha adequada para o levantamento de atributos.

\section{TREINAMENTO DA EQUIPE}

Após cada provador ter levantado os termos descritivos para os pares de amostras, a equipe se reuniu e discutiu os termos levantados. Nesta etapa, os termos que expressaram o mesmo significado foram agrupados em um só atributo. Já os 
termos poucos utilizados pelos provadores foram eliminados. No final das sessões, foi gerada uma lista de termos descritivos (Tabela 2) com os respectivos extremos da escala utilizada (Figura 1). Durante o treinamento, os provadores foram solicitados a avaliar a intensidade de cada atributo sensorial das amostras.

Foi utilizada uma escala não estruturada de nove centímetros ancorada nos extremos com termos definidos pela equipe (Figura 1). As amostras foram apresentadas monadicamente e a ordem de apresentação foi balanceada. Os testes foram realizados em cabines individuais adaptadas visando manter o isolamento de cada provador. Os provadores utilizaram água mineral para lavar o palato entre uma amostra e outra.

\section{SELEÇÃO DOS PROVADORES}

Após o treinamento, os provadores avaliaram as amostras, com três repetiçôes, utilizando a ficha desenvolvida. Os provadores foram selecionados em função das habilidades de discriminar as amostras e repetibilidade nas avaliações. Os provadores que apresentaram probabilidade de $\mathrm{F}_{\text {amostras }}$ não significativo $(\mathrm{p} \geq 0,05)$, ou $\mathrm{F}_{\text {repeti̧̧a }}$ significativo $(\mathrm{p} \leq 0,05)$, em mais que um atributo.

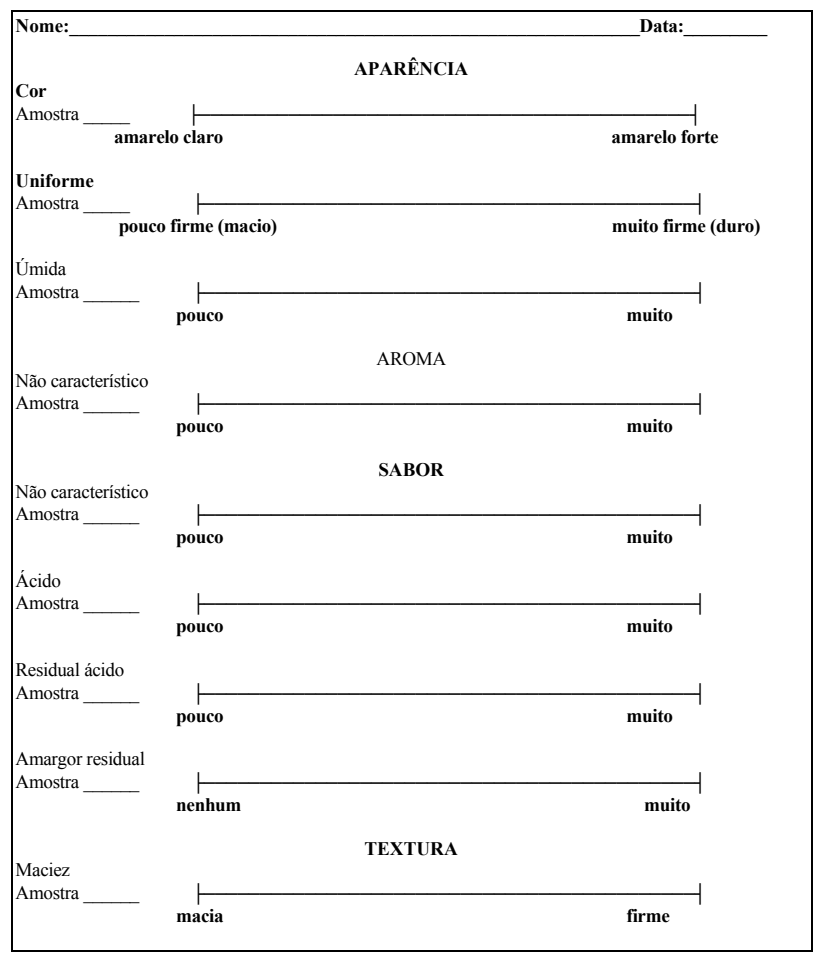

Figura 1 - Ficha de avaliação utilizada para analise sensorial descritiva dos palmitos.

\section{ANALISE DAS AMOSTRAS}

As amostras das três marcas comerciais de palmito foram avaliadas em triplicata pelos provadores selecionados, em relação aos atributos mostrados na Tabela 2 , nas mesmas condições que as citadas no treinamento dos provadores.

Tabela 2 - Definição dos termos descritivos.

\begin{tabular}{|c|c|}
\hline Atributos & Definições \\
\hline Cor amarela & $\begin{array}{l}\text { Refere-se à intensidade da cor amarelada do } \\
\text { palmito. }\end{array}$ \\
\hline Uniformidade da cor & $\begin{array}{l}\text { Refere-se à tonalidade amarelada em todo } 0 \\
\text { palmito. }\end{array}$ \\
\hline Úmida & $\begin{array}{l}\text { Refere-se à aparência do produto de conter água } \\
\text { em seu interior. }\end{array}$ \\
\hline $\begin{array}{l}\text { Aroma não } \\
\text { característico }\end{array}$ & $\begin{array}{l}\text { Refere-se ao aroma característico do palmito } \\
\text { tradicional. }\end{array}$ \\
\hline $\begin{array}{l}\text { Sabor não } \\
\text { característico }\end{array}$ & $\begin{array}{l}\text { Refere-se ao sabor característico de palmito } \\
\text { tradicional. }\end{array}$ \\
\hline Gosto ácido & Refere-se à presença gosto ácido do palmito. \\
\hline Sabor residual ácido & $\begin{array}{l}\text { Refere-se ao gosto ácido que permanece por um } \\
\text { tempo após degustação. }\end{array}$ \\
\hline Amargor final & $\begin{array}{l}\text { Refere-se ao gosto amargo percebido ao final da } \\
\text { degustação. }\end{array}$ \\
\hline Maciez & $\begin{array}{l}\text { Refere-se à intensidade de força aplicada pelos } \\
\text { dentes ao morder. }\end{array}$ \\
\hline
\end{tabular}

\section{ANÁLISE ESTATÍSTICA}

Os dados obtidos na análise sensorial foram analisados através da análise de variância (ANOVA) utilizando o programa estatístico SAS (1989) e, tendo sido detectadas diferenças significativas entre as médias $(\mathrm{p} \leq 0,05)$, as mesmas foram submetidas ao teste de Tukey.

\section{RESULTADOS E DISCUSSÃO}

De acordo com os resultados obtidos, pode-se verificar através da Figura 2 e da Tabela 3, as diferenças entre os palmitos estudados. Os atributos sensoriais que descreveram os palmitos de pupunha foram: aparência: cor amarela, uniformidade da cor e aparência úmida; aroma: não característico; sabor: não característico, gosto ácido, sabor residual ácido, amargor final e textura: maciez.

Nove provadores foram excluídos da equipe por não apresentaram capacidade de discriminação dos atributos avaliados ou repetibilidade nas avaliaçōes em mais de dois atributos.

A análise de variância nos dados sensoriais para cada atributo indicou diferença significativa ao nível de 5\% para seis dos termos descritivos estudados pelos onze provadores selecionados, e são apresentadas na Tabela 3. 


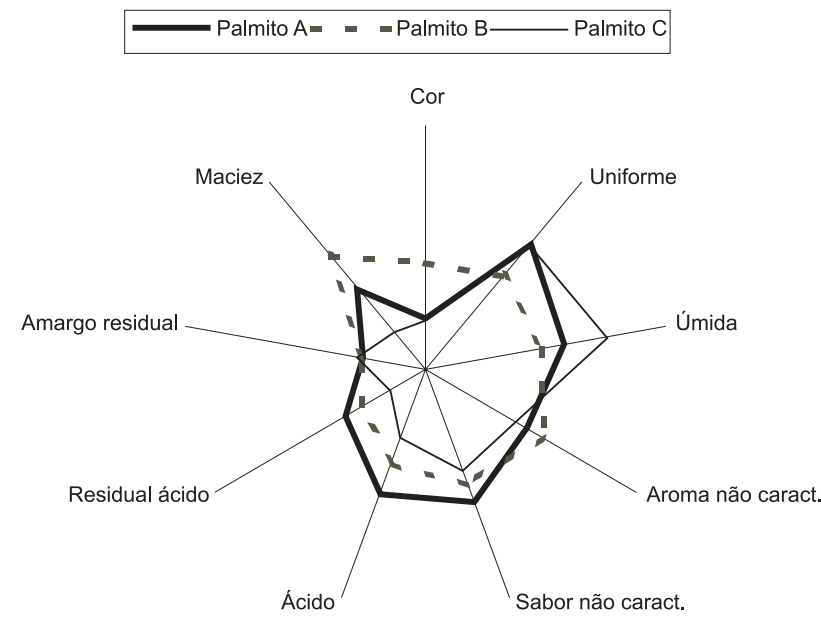

Figura 2 - Perfil sensorial das amostras de palmito de pupunha estudados.

Tabela 3 - Média dos atributos sensoriais avaliados nas amostras de palmito.

\begin{tabular}{llll}
\hline ATRIBUTOS & PALMITO A & PALMITO B & PALMITO C \\
\hline APARÊNCIA & & & \\
Cor amarela & $1,86 \mathrm{~b}$ & $3,93^{\mathrm{a}}$ & $1,79 \mathrm{~b}$ \\
Uniforme & $6,06 \mathrm{a}$ & $4,56 \mathrm{~b}$ & $5,98 \mathrm{ab}$ \\
Úmida & $5,15 \mathrm{~b}$ & $4,35 \mathrm{~b}$ & $6,78 \mathrm{a}$ \\
\hline AROMA & & & \\
Não Característico & $4,31 \mathrm{a}$ & $4,96^{\mathrm{a}}$ & $3,80 \mathrm{a}$ \\
\hline SABOR & & & \\
Não Característico & $5,20 \mathrm{a}$ & $4,50^{\mathrm{a}}$ & $3,95 \mathrm{a}$ \\
\hline Ácido & $4,89 \mathrm{a}$ & $3,64 \mathrm{~b}$ & $2,65 \mathrm{~b}$ \\
Residual ácido & $3,40 \mathrm{a}$ & $2,71 \mathrm{ab}$ & $1,50 \mathrm{~b}$ \\
\hline Amargor final & $2,30 \mathrm{a}$ & $2,42^{\mathrm{a}}$ & $2,56 \mathrm{a}$ \\
\hline TEXTURA & & & \\
\hline Maciez & $3,87 \mathrm{~b}$ & & \\
& $5,45 \mathrm{a}$ & $5,45^{\mathrm{a}}$ & $1,82 \mathrm{c}$ \\
\hline
\end{tabular}

Médias na mesma linha seguidas de letras iguais não diferem significativamente $(p \geq 0,05)$.

Através dos resultados verificou-se que a amostra de palmito $\mathrm{B}$ apresentou coloração amarelada mais intensa que as amostras $\mathrm{A} e \mathrm{C}$, que não diferiram significativamente entre si ( $\mathrm{p} \geq 0,05)$. De acordo com o Kapp et al. (2003), o pigmento amarelado é característico do palmito de pupunha.

Verruma-Bernardi et al. (2003) estudando a aceitabilidade do palmito de pupunha picados e em rodelas, mostraram que apesar do palmito de pupunha não ter a cor e o sabor convencionais para o palmito a que se está acostumado, ele foi relativamente bem aceito.
Em relação a uniformidade da cor a amostra de palmito A apresentou cor significativamente mais uniforme que amostra de palmito B. Já a amostra de palmito $\mathrm{C}$ possui uniformidade de cor intermediária entre as amostras A e B sem diferir significativamente delas.

Ferreira et al. (1981; 1982a) verificaram que o palmito de pupunha em relação ao palmito juçara apresentou cor amarelo claro creme uniforme, não esperado para o palmito ao qual se está habituado.

Em relação à aparência úmida verificou-se que a amostra de palmito $C$ diferiu significativamente $(p \leq 0,05)$ das amostras das amostras de palmito $\mathrm{A}$ e $\mathrm{B}$, as quais não diferiram entre si, apresentando aparência mais úmida.

Para os atributos aroma e sabor não característicos e amargor final as amostras de palmito não apresentaram diferença significativa entre si $(\mathrm{p} \geq 0,05)$.

Os resultados da análise de sabor mostraram que em relação aos atributos de

$\mathrm{Na}$ percepção do gosto ácido a amostra de palmito A diferiu significativamente das demais amostras, apresentando gosto ácido mais intenso que as amostras de palmito B e C.

Em estudo realizado por Ferreira et al. (1981/1982b), onde foram comparados palmitos pupunha e juçara em relação às características físicas e químicas verificou-se que o palmito de juçara apresenta maior poder tampão que o de pupunha; as concentrações de açúcares redutores e totais do palmito de pupunha foram maiores (praticamente de 2 a 3 vezes, respectivamente) do que no palmito juçara. Os autores relatam que sem dúvida, estes teores devem ter influência nas diferenças de sabor entre os palmitos.

Na percepção de sabor residual ácido foi observado que a amostra de palmito A possui sabor residual ácido mais acentuado do que e a amostra de palmito $\mathrm{C}$, enquanto que a amostra B foi classificada de modo intermediário sem diferir estatisticamente das demais amostras.

Uzelac et al. (1976) concluíram que o sabor amargo em palmito pode ter sua origem em compostos fenólicos e também, provavelmente, as quantidades daqueles compostos e a natureza das suas estruturas químicas, como também a outros componentes como aminoácidos livres e peptídeos.

Segundo Soares (1997), a pupunha é uma espécie de palmito que apresenta algumas características próprias e muito distintas tais como: ausência de escurecimento enzimático, textura macia e sabor característico ligeiramente adocicado.

Outros estudos descritos por Ferreira et al. (1981/1982a) mostram que o palmito de pupunha se caracteriza pelo sabor mais doce e coloração mais amarelada e apresenta vantagens industriais e agronômicas, quando comparada com outras espécies. Tem baixos índices de substâncias oxidantes, o que 
proporciona alterações mínimas no sabor e aroma após o beneficiamento, bom sabor, textura firme e macia.

A análise estatística sobre a maciez das amostras mostrou que as três amostras de palmito diferiram significativamente entre si $(p<0,05)$, possuindo a amostra de palmito $C$ textura menos firme (macia) e a amostra de palmito $\mathrm{B}$ textura mais firme.

Apesar da diferença significativa em mais da metade dos atributos levantados (cor amarelada, aparência macia, aparência úmida, gosto ácido e maciez), os provadores sempre tiveram a tendência de utilizar a mesma parte da escala para pelo menos 8 atributos, porem para aparência macia e textura macia os provadores utilizaram partes mais distantes da escala.

Segundo Ferreira et al. (1981/1982a) e Verruma-Bernardi, et al. (2003), o palmito de pupunha tem apresentado boa aceitação no mercado, sendo capaz de competir com palmitos das palmeiras do gênero Euterpe.

\section{CONCLUSÕES}

O uso da Análise Descritiva Quantitativa, forneceu informaçōes do levantamento de atributos sensoriais para a discriminação das amostras de palmito de pupunha;

Os resultados mostraram que houve diferenças significativas em 6 atributos entre as 3 marcas testadas;

Os provadores descreveram os palmitos de pupunha como: cor amarela clara, aparência uniforme e úmida, aroma e sabor não característicos e sabor ácido com valores intermediários, residual acido e amargor final com valores de pouco e textura muito macia a uma textura intermediária.

\section{BIBLIOGRAFIA CITADA}

Araújo, I.C. 1996. Potencialidade da pupunheira: uma visão do ponto de vista do agrobusiness. In: Workshop sobre as culturas de cupuaçu e pupunha na Amazônia, 1, Manaus. Anais... Manaus: EMBRAPA-CPAA.

Bovi, M.L.A. 1998. Palmito pupunha: informaçôes básicas para cultivo. Boletim Técnico, 173, Campinas. IAC. 50pp.

Ferreira, V.L.P.; Graner, M.; Bovi, M.L.A; Draetta, I.S.; Paschoalino, J,E.; Shirose, I. 1981/1982a. Comparação entre os palmitos de Guilielma gasipaes Bailey (pupunha) e Euterpe edulis Mart (juçara). I Avaliações químicas, físicas, organolépticas e bioquímicas. Coletânea do Instituto de Tecnologia de Alimentos, Campinas. v.12. p. 255-272.
Ferreira, V.L.P.; Graner, M.; Bovi, M.L.A.; Figueiredo, J.B.; Angelucci, E.; Yokomizo, Y. 1981.1982b. Comparação entre os palmitos das palmeiras Giulielma gasipaes Bayley (pupunha) e Euterpe edulis Mart. (juçara). II Avaliações físicas e químicas. Coletânea do Instituto de Tecnologia de Alimentos, Campinas. v. 12. p. 273-282.

Ferreira, V.L.P.; Bovi, M.L.A., Carvalho, C.R.L. et al. 1990. Composição química e curvas de titulação de acidez do palmito de pupunha (Bactris gasipaes H.B.K.) de diversas localidades. Coletânea do Instituto de Tecnologia de Alimentos, Campinas. v.20, p. 96-104.

Freitas, R.J.S., Fugman, H.A.J. 1990. Componentes minerais do palmito (Euterpe edulis Mart.). Boletim do CEPPA, 8(1): 3539.

Kapp, E.A.; Pinheiro, J.L.; Raupp, D.S. et. al. 2003. Tempo de preservação de tolete de palmito pupunha (Bactris gasipaes) minimamente processado e armazenado sob refrigeração. Publ. UEPG Ci. Exatas Terra, Ci. Agr. Eng., 9(3): 51-57.

Moskowitz, H.R. 1983. Product testing and sensory evalution offoods. Westport:. Food \& Nutrition Press. 605pp.

Pantoja, L.; Maeda, R.N.; Andrade, J.S.; Pereira Jr., N.; Carvalho, S.M.S.C.; Astolfi-Filho, S. 2000. Bebida alcoólica fermentada a partir de pupunha (Bactris gasipaes Kunt). In: Anais do IX Congresso Brasileiro de Ciência e Tecnologia de Alimentos. Fortaleza-CE. v.4. p.99.

SAS INTITUTE INC. 1989. SAS/STAT; user's guide: version 6, $4 .$. Ed.Cary, SA,. v.2, 846pp.

Soares, A.G. 1997. Palmito de pupunha - Alternativas de processamento. Revista de Horticultura Brasileira, Brasília. v.15. p. 198-199.

Stone, H.S.; Sidel, J.L. 1985. Sensory evalution practices. London: Academic Press. 311pp.

Uzelac, M.; Abdala, C.; Franco, J.F. 1976. Estudos comparativos entre os palmitos de sabor doce (palmito Juçara - Euterpe edulis Mart. e Açaí - Euterpe olerácea Mart) e de sabor amargo (Guariroba - Syagrus olerácea Becc). II Compostos fenólicos. Coletânea do Instituto de Tecnologia de Alimentos, Campinas, 7(2): 371-383.

Verruma-Bernardi, M.R.; Cavalcanti, A.C.D.; Kajishima, S. 2003. Aceitabilidade do palmito de pupunha. Boletim do CEPPA, 21(1): 121-130

Villachica, H. 1996. Cultivo del pijuayo (Bactris gasipaes Kunth.) para palmito en la Amazonia. Lima, Peru: FAO/Tratado de Cooperacion Amazonica, 153pp.

Recebido em 27/10/2004

Aceito em 14/09/2007 
